\title{
FINITELY NONREFLEXIVE BANACH SPACES
}

\author{
MAREK WÓJTOWICZ
}

(Communicated by William J. Davis)

\begin{abstract}
A Banach space $X$ is said to be finitely nonreflexive if for some $n \geq 1$ the space $H_{n}(X)$ is reflexive with $\operatorname{dim} H_{n}(X) \geq 1$, where $H_{0}(X)=X$, and $H_{n+1}(X)=H_{n}(X)^{* *} / H_{n}(X)$. A simple construction of (pairwise nonisomorphic) finitely nonreflexive separable Banach spaces which are both nonisomorphic to their Cartesian squares and isomorphic to their biduals is presented.
\end{abstract}

\section{INTRODUCTION}

In 1950 R. C. James [8] constructed the first example of a Banach space $J$ isomorphic to its second conjugate and nonreflexive: $\operatorname{dim}\left(J^{* *} / J\right)=1$. As proved by Civin and Yood [5] each quasi-reflexive space (i.e., of finite codimension in its bidual) is isomorphic to $Y^{* *}$ for some quasi-reflexive space $Y$. James' construction has been modified in many ways to obtain examples connected with both the structure of the space $X^{* *} / X$ for $X$ a Banach space (see $[2,9,11,12])$ and the duals of $X[2,12]$. Moreover, Bessaga and Petczyński [4] observed that each quasi-reflexive space is nonisomorphic to its Cartesian square (infinitely dimensional reflexive spaces with the same property have been constructed by Figiel [7], and Bellenot [3]). The notion of finitely nonreflexive Banach spaces, as a generalization of quasi-reflexive spaces, has been considered by Bellenot [1, 2], who was inspired by some results of Davis, Johnson, and Lindenstrauss [6]; a similar concept can be found in [13], p. 249.

In the paper [12], for $X$ an arbitrary Banach space, the natural analogue $J(X)$ of the space $J$ has been constructed and studied. Let us define the sequence of Banach spaces $\left(J_{n}(X)\right)_{n=1}^{\infty}$ by $J_{l}(X)=J(X)$, and $J_{n+1}(X)=$ $J\left(J_{n}(X)\right)$. In this note we shall show that if $\operatorname{dim} X$ is finite or $X$ is the Figiel's space [7], then all the mentioned-above properties of the quasi-reflexive space

Received by the editors April 20, 1988 and, in revised form, July 14, 1988.

1980 Mathematics Subject Classification (1985 Revision). Primary 46B10; Secondary 46B25.

Key words and phrases. Biduals of Banach spaces, quasi-reflexive spaces. 
$J$ are heredited by (separable and pairwise nonisomorphic) finitely nonreflexive spaces $J_{n}(X), n \geq 1$.

\section{Preliminaries}

Our terminology and notation and standard (e.g., as in [10]). We write $X \approx$ $Y$ or $X \cong Y$ if the Banach spaces $X$ and $Y$ are isomorphic or isometric, respectively. $X$ is said to be 1-complemented in $Y$ if there exists of a norm 1 projection $P$ in $Y$ such that $X \cong P Y . X^{k}$ denotes the Cartesian product of $k$-copies of $X$. Let $\pi_{X}$ denote the canonical embedding of $X$ into $X^{* *}$. We shall identify $X$ with $\pi_{X}(X)$. We define $H_{0}(X)=X, H(X)=X^{* *} / X$, and inductively, $H_{n}(X)=H\left(H_{n-1}(X)\right) . \quad X$ is said to be $n$-reflexive [resp. quasi-reflexive of order $m$ ( $m$ finite and $\geq 1)$ ] if $H_{n}(X)$ is reflexive with $\operatorname{dim} H_{n}(X) \geq 1$ (see [2], p. 105) [resp. $H(X)$ is of dimensional $m$ ]; then $n$ is called the reflexive dimension of $X$ ([13], p. 249). $X$ is said to be finitely nonreflexive if $X$ is $n$-reflexive for some $n \geq 1$. All quasi-reflexive spaces are 1-reflexive, but there exist non-quasi-reflexive spaces, which are 1-reflexive ([2, $11,12])$. General properties of finitely nonreflexive Banach spaces have been studied in $[2,6,13]$. Let $X=(X,\|\|)$ be an arbitrary Banach space. Then the Banach space $\widetilde{J}(X)=(\widetilde{J}(X),\|\|)$ is defined as follows [12]:

$$
\widetilde{J}(X)=\left\{x=\left(x_{i}\right) \in X^{\mathbf{N}}:\|x\|=\sup _{p \in \mathscr{P}} \sigma_{\rho}(x)<\infty\right\},
$$

where $\mathscr{P}$ is the set of all strictly increasing sequences $p=(p(1), \ldots, p(2 k+1))$ in $\mathbf{N}, k \in \mathbf{N} \cup\{0\}$, and

$$
\sigma_{p}(x)=\left(\sum_{i=1}^{k}\left\|x_{p(2 i)}-x_{p(2 i-1)}\right\|^{2}+\left\|x_{p(2 k+1)}\right\|^{2}\right)^{1 / 2} .
$$

It is easily seen that for each $x=\left(x_{i}\right) \in \widetilde{J}(X)$ the limit $x_{\infty}=\lim _{i \rightarrow \infty} x_{i}$ exists in $X$. The James space $J(X)=(J(X),\|\| \|)$ is the closed subspace of $\widetilde{J}(X)$ consisting of those $x \in \widetilde{J}(X)$ for which $x_{\infty}=0$, and is isomorphic to the $J$-sum of $\left(X_{n}\right)_{n=1}^{\infty}$ with $X_{n}=X$ constructed by Bellenot ([2], Remark 7) (notice that $J=J(\mathbf{R})$ ). The subspace $[X]$ of all constant sequences is isometric in a natural manner to $X$ and is the range of the norm 1 projection $Q$ in $\widetilde{J}(X)$ defined by $Q(x)=\left(x_{\infty}, x_{\infty}, \ldots\right)$. Moreover, $\operatorname{ker} Q=J(X)$. Hence $\widetilde{J}(X)=J(X) \oplus[X]$. By an argument similar to that of [8], $J(X) \oplus[X] \approx J(X)$.

Since $J(X)$ has a shrinking Schauder decomposition of a sequence spaces each isometric to $X$, by a straightforward generalization of Proposition 1.b.2 [10, p. 8], the space $J(X)^{* *}$ is isometric to $\widetilde{J}\left(X^{* *}\right)$ via the mapping $F \mapsto\left(F_{n}\right)$, where $\left(F_{n}\right)$ is the uniquely determined sequence in $X^{* *}$ such that $F(f)=$ $\sum F_{n}\left(f_{n}\right)$ holds for all $f=\left(f_{n}\right) \in J(X)^{*}$ (cf. [12]; Theorem, p. 185). It follows that

$$
J(X)^{* *} \cong J\left(X^{* *}\right) \oplus\left[X^{* *}\right] \approx J\left(X^{* *}\right)
$$


and if $X$ is reflexive, then

$$
J(X)^{* *} / J(X) \approx X
$$

Note that " $H$ " and " $J$ " "operators" are functors. We have $T: X \rightarrow Y$ induces the functors $H(T): H(X) \rightarrow H(Y)$ and $J(T): J(X) \rightarrow J(Y)$ defined by $H(T)\left(x^{* *}+X\right)=T^{* *}\left(x^{* *}\right)+Y$ and $J(T)\left(x_{i}\right)=\left(T x_{i}\right)$, which preserve the addition of linear operators. These functorial properties easily imply $H(X \oplus Y) \approx H(X) \oplus H(Y)$ and $J(X \oplus Y) \approx J(X) \oplus J(Y)$. Obviously " $J$ " preserves isomorphisms (onto) and, by ([13]; Theorems 1.1 and 1.2), the same holds for " $H$ ". In particular, any two isomorphic finitely nonreflexive Banach spaces have the same reflexive dimension.

\section{Construction}

We shall now present our main result.

Theorem. Let $\{0\} \neq X$ be a reflexive Banach space. Put $Z_{n}=J_{n}(X), n \geq 1$. Then for all integers $n, m$ we have:

(i) $Z_{n}$ is isomorphic to $Z_{n}^{* *}$;

(ii) $Z_{n}$ is $n$-reflexive; more precisely, $H_{n}\left(Z_{n}\right) \approx X^{n !}$;

(iii) iff $n<m$, then $Z_{n}$ is nonisomorphic to $Z_{m}$ and 1-complemented in $Z_{m}$.

Moreover, if $X^{n !}$ is nonisomorphic to $X^{2 n !}$, then

(iv) $Z_{n}$ is nonisomorphic to $Z_{n} \oplus Z_{n}$.

If $X$ is separable, then $J(X)$ is separable also (since $\left\|\left(x_{i}\right)\right\|^{2} \leq 2 \sum\left\|x_{i}\right\|^{2}$ holds for each $\left.\left(x_{i}\right) \in J(X)\right)$. Hence the corollary below is an immediate consequence of the theorem.

Corollary. Let the sequence $\left(J_{n}\right)_{n=1}^{\infty}$ of separable Banach spaces be defined by the formula $J_{n}=J_{n}(\mathbf{R})$. Then for all integers $n<m$ we have:

(i) $J_{n}$ is isomorphic to $J_{n}^{* *}$ and nonisomorphic to $J_{n} \oplus J_{n}$;

(ii) $J_{n}$ is n-reflexive; more precisely, $H_{n-1}\left(J_{n}\right)$ is quasi-reflexive of order $n !$;

(iii) $J_{n}$ is nonisomorphic to $J_{m}$ and 1-complemented in $J_{m}$.

Remark. The reflexive and separable Banach space $\mathbf{F}$ constructed by Figiel [7] is "close" to $\mathbf{R}: \mathbf{F}^{k} \approx \mathbf{F}^{1}$ iff $k=l$ (in particular, $\boldsymbol{F}$ is nonisomorphic to $\mathbf{F}^{2}$ ), and of course each of the spaces $\mathbf{F}^{k}, k=1,2, \ldots$, is of this type. Using Theorem (ii) it is easily seen that $J_{n}\left(\mathbf{F}^{k}\right) \approx J_{n}\left(\mathbf{F}^{1}\right)$ [and $J_{n}\left(\mathbf{R}^{k}\right) \approx J_{n}\left(\mathbf{R}^{\mathrm{l}}\right)$ ] iff $k=l$ and $n=m$; hence the sequences of Banach spaces $\left(J_{n}\left(\mathbf{F}^{k}\right)\right)_{n=1}^{\infty}$ [and $\left(J_{n}\left(\mathbf{R}^{k}\right)\right)_{n=1}^{\infty}$, $k=1,2 \ldots$, have properties given in the corollary with $H_{n-1}\left(J_{n}\left(\mathbf{F}^{k}\right)\right)$ nonquasi-reflexive [and $H_{n-1}\left(J_{n}\left(\mathbf{R}^{k}\right)\right)$ quasi-reflexive of order $k n$ !] and contain no pairwise isomorphic elements. 
Proof of the theorem. (i) By (1), $Z_{1}^{* *}=J(X)^{* *} \approx J(X)=Z_{1}$, and inductively, using the fact that $Y \approx Y^{* *}$ implies $J(Y) \approx J\left(Y^{* *}\right), Z_{n}^{* *} \approx Z_{n}$ for all integers $n$.

(ii) We shall prove that the relations

$$
\begin{gathered}
Z_{n}^{* *}=Z_{n} \oplus V_{n}, \text { where } V_{n} \approx\left(Z_{n-1}\right)^{n}, \\
H_{n}\left(Z_{n}\right) \approx X^{n !},
\end{gathered}
$$

hold for all $n \in \mathbf{N}$. If $n=1$, then by (1) and (2), $Z_{1}^{* *}=Z_{1} \oplus V_{1}$, where $V_{1}=[X] \cong Z_{0}^{l}$, and $H_{1}\left(Z_{1}\right) \approx X^{l}$. If $n=k+1$, then (1) follows that $Z_{k+1}^{* *}=J\left(Z_{k} \oplus V_{k}\right) \oplus\left[Z_{k}^{* *}\right] \cong Z_{k+1} \oplus V_{k+1}$, where $V_{k+1}=J\left(V_{k}\right) \oplus\left[Z_{k}^{* *}\right]$. Since $Z_{k}^{* *} \approx Z_{k}$, we have $V_{k+1} \approx J\left(\left(Z_{k-1}\right)^{k}\right) \oplus Z_{k} \approx\left(Z_{k}\right)^{k+1}$; it proves (3). To prove (4) observe that $X \approx Y$ implies $H_{n}(X) \approx H_{n}(Y)$ for all integers $n$, so by (3) we get $H_{k+1}\left(Z_{k+1}\right)=H_{k}\left(H\left(Z_{k+1}\right)\right) \approx H_{k}\left(Z_{k}^{k+1}\right) \approx H_{k}\left(Z_{k}\right)^{k+1}$.

(iii) Since the reflexive dimension is invariant under isomorphisms, by the just proved statement (ii) the first part of (iii) is obvious. The second one follows from the fact that for each integer $n$ the space $Z_{n}$ is isometrically isomorphic to the 1-complemented subspace of $Z_{n+1}$ of the form

$$
\left\{\left(x_{i}\right) \in J\left(Z_{n}\right): x_{i}=0 \text { for all } i \geq 2\right\} \text {. }
$$

(iv) $J(X \oplus Y) \approx J(X) \oplus J(Y)$ implies that $Z_{n} \oplus Z_{n} \approx J_{n}(X \oplus X)$, thus (4) follows that if $Z_{n} \approx Z_{n} \oplus Z_{n}$, then $X^{n !} \approx X^{2 n !}$.

\section{ACKNOWLEDGMENT}

I am indebted to the referee for his very helpful remarks.

\section{REFERENCES}

1. S. F. Bellenot, Uniformly complemented $l_{p}^{n}$ 's in quasi-reflexive Banach spaces, Israel J. Math. 39 (1981), 234-246.

2. _ The J-sum of Banach spaces, J. Funct. Anal. 48 (1982), 95-106.

3. _ Tsirelson superspaces and $l_{p}$, J. Funct. Anal. 69 (1986), 207-228.

4. Cz. Bessaga and A. Pełczyński, Banach spaces non-isomorphic to their Cartesian squares. I, Bull. Polish Math. Acad. Sci. 8 (1960), 77-80.

5. P. Civin and B. Yood, Quasi-reflexive spaces, Proc. Amer. Math. Soc. 9 (1957), 906-911.

6. W. J. Davis, W. B. Johnson and J. Lindenstrauss, The $l_{1}^{n}$-problem and degrees of non-reflexivity, Studia Math. 55 (1974), 311-317.

7. T. Figiel, An example of an infinite dimensional reflexive Banach space non-isomorphic to its Cartesian square, Studia Math. 42 (1972), 295-306.

8. R. C. James, Bases and reflexivity of Banach spaces, Ann. of Math. 52 (1950), 518-527.

9. J. Lindenstrauss, On James' paper "Separable conjugate spaces", Israel J. Math. 9 (1971), 279284.

10. J. Lindenstrauss and L. Tzafriri, Classical Banach spaces I, Springer-Verlag, Berlin, 1977.

11. P. V. Semenov, James-Orlicz spaces, Uspekhi Mat. Nauk, 34 (1974), 209-211 (in Russian). 
12. M. Wójtowicz, On the James space $J(X)$ for a Banach space $X$, Ann. Soc. Math. Polon., Ser. I Comment. Math. Prace Mat. 23 (1983), 183-188.

13. K.-W. Yang, The reflexive dimension of an R-space, Acta Math. Hungar. 35 (1980), 249-255.

Pedagogical University, Institute of Mathematics, Pl. Slowiański 6, 65-069 Zielona Góra, Poland 